\title{
16
}

\section{Design of Multimedia Global PACS CORBA Environment}

\author{
Dr. Ralph Martinez \& Sheau-Ling Hsieh \\ University of Arizona \\ Dept. of Electrical \& Computer Engineering, University of Arizona, \\ Tucson, AZ 85719, USA. Telephone: (520)621-6174. Fax: \\ (520)621-3862. email: martinez, slhsieh@ece.arizona.edu
}

\begin{abstract}
In this paper, we present an approach to developing Global Picture Archiving and Communication System (Global PACS) applications based on the Common Object Request Broker Architecture (CORBA) specification. Designing distributed Global PACS applications, based on the CORBA specification, will feature an open, heterogeneous, and scalable architecture. It will also make the Global PACS remote consultation and diagnosis, including synchronized image annotation, voice and video, technologically independent. The applications can communicate through various networks as long as they support CORBA components. The paper presents the initial development and the basic functions of remote consultation sessions have designed based on the CORBA definitions. Further research and development work is summarized.
\end{abstract}

\section{Keywords}

remote consultation and diagnosis, database management system, database archive system, viewing workstations, object-oriented distributed computing, client-server architecture.

\section{INTRODUCTION}

A global picture archiving and communication system (Global PACS) operates in a medical telecommunication environment (Martinez 1992). It manages patients' digital images and laboratory results over a widespread geographical area. The Global PACS infrastructure is a highly multi-vendor environment. It consists of vest networks of heterogeneous, autonomous, and distributed computing resources, including computers, acquisition equipment, as well as multimedia data (files and database). This advanced telemedicine system gives hospitals a strategic edge in achieving cost-effective, quality patient care, and a long-term, comprehensive solutions for today's as well as tomorrow's health care and medical information needs.

To date, we have developed real-time, $\mathrm{X}$-Window based medical diagnosis and remote consultation on RISC and PC-based workstations (Martinez 1992-Martinez 1995). The 
consultation has synchronized voice and image annotation features. The communication used in the Global PACS network is based on the TCP/UDP sockets, remote procedure calls (RPCs), as well as file transfer (FTP). We also developed a distributed file system for multimedia patient information archiving.

In this paper, we present our approach using the Common Object Request Broker Architecture (CORBA) (OMG 1991) to modify and enhance the current Global PACS applications. In other words, we plan to migrate the Global PACS applications into an object-oriented environment to provide interoperability and scalability. Ultimately, this concept of distributed object-oriented Global PACS architecture is intended to support capabilities including (Manola 1992):

- The ability to integrate heterogeneous and multimedia data types, including both conventional formatted data and unconventional data types, i.e., sound and image data, as well as patient records.

- The ability to integrate resources at whatever level of granularity is appropriate, including multimedia objects that represent entire remote consultation workstations or patient database systems, as well as objects that represent individual application abstractions or data items such as X-ray images.

- The ability to invoke and synchronize the execution of combinations of objects anywhere in the integrated Global PACS network, and to move data or objects as necessary, in response to a given request.

- The ability to support cooperation among intelligent components, i.e, computer controlled imaging equipments or microscopes.

The remainder of the paper is organized as follows. Section 2 in this paper summarizes the Global PACS environment and its functionalities. Section 3 discusses the current problems and possible solution while migrating into the object-oriented environment. Section 4 provides a detailed mapping of the current Global PACS software architecture into the CORBA environment. Section 5 contains conclusion and possible future work.

\section{GLOBAL PACS BACKGROUND}

A Global PACS is a national network which interconnects several PACS networks at medical and hospital complexes using the national backbone network. A complete Global PACS system with multiple interconnected PACSs and its major components is shown in Figure 1 (Martinez et al. 1994). The components include: imaging equipment for acquiring images, viewing workstations for consultation, database management system (or database archive system) for storage, retrieval patient information, and communication network for connecting all of these components into a integrated system. The global backbone network in Figure 1 is currently the Internet.

The Global PACS environment enables joint collaboration between pathologists, radiologists, or physicians while they are in different geographical locations. One method of collaboration is to allow radiologists to view the same image folder from different sites, i.e., Local and Remote, so that a diagnosis can be performed. In a remote consultation and diagnosis (RCD), a set of patient images are requested from a database archive system (DBAS) and sent to, displayed at both Local and Remote sites through the Global PACS 


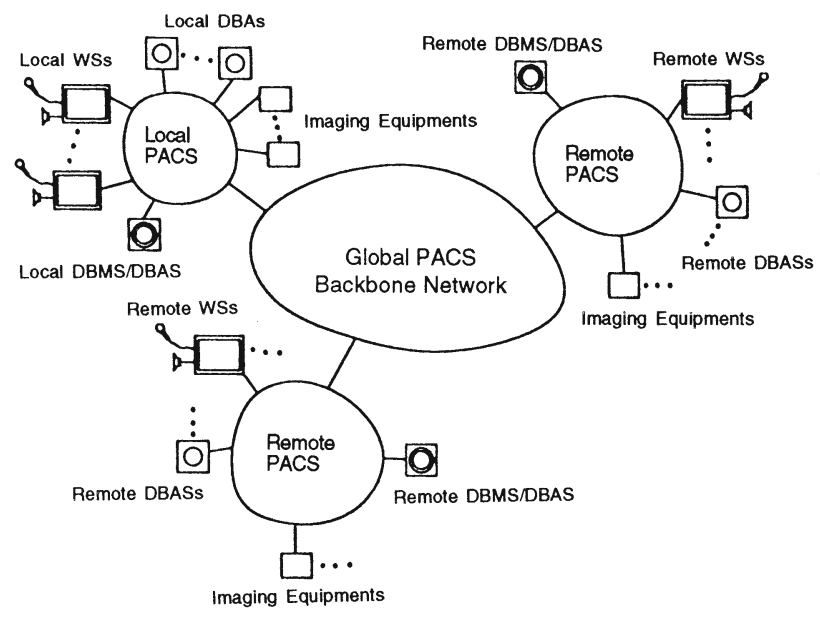

Figure 1 Global PACS Environment.

networks. Each operation can be digitally recorded and replayed later for future study and analysis. The Global PACS applications support real-time, image, audio/voice, and compressed video cor. munications for medical diagnosis and consultation. A RCD system with a Serial Line Internet Protocol (SLIP) connection has been developed for rural areas which have no direct Internet connections (Martinez et al. 1995).

A Global PACS software architecture and its collaborating modules are described as follows.

\subsection{Database in Global PACS}

Each PACS has one database management system (DBMS) which manages all the data/file domains in a PACS. Each database archive system (DBAS) accesses one or more domains within a PACS. Therefore, a DBMS manages DBAS(s) in a PACS. Sometimes, a DBMS and a DBAS reside on the same node. This node is represented as a DBMS/DBAS machine.

DBMS manages user's queries and passes the requests to DBAS(s). Each DBAS stores and retrieves patient's folders as inquired. A patient can have one or more folders. Each folder contains several image sets; each image set has 5-7 image files. The image files are in the form of Graphics Interchange Format (GIF) or in raw image data. Images can also have a DICOM format (ACR-NEMA 1992).

During a RCD session, a DBAS also stores the study session into files for later replay or future analysis. The study sessions are subdivided further into patient's records and are called events. If the study session is for an individual patient, the information files 
are stored under his/her patient ID. If the study session is related to a disease, particular area of interests or conference, the data files are stored under the event.

\subsection{DBMS/DBAS Functional Modules}

Functionally, a DBAS contains two modules, i.e., DBAS manager and DBAS storage server. The DBAS manager is a daemon process which does the data communication and network interface. The DBAS storage server provides image/data files storage and retrieval. It receives requests from the DBAS manager and responds with the inquired actions.

In a DBMS/DBAS machine, it has three modules: DBMS manager, DBMS data server, and DBAS storage server. The DBMS manager is a daemon process which does the data communication and network interface. The DBMS data server responds forwarded queries from the DBMS manager and searches the database directory lists in the PACS. This module is not present in the DBAS only host. The DBAS storage server provides the same functions as in the DBAS module.

Both DBAS manager and DBMS manager interface with the Local and Remote viewing workstations for remote consultation and diagnosis. They deliver the selected images to the Local and Remote workstations.

\subsection{Viewing Workstations}

The basic remote consultation scenario begins at a Local workstation. The Local workstation sends a request to a Remote workstation for a consultation. In the meantime, the Local workstation also requests an image file to be displayed on both workstations from an associated DBAS. The Remote workstation responds with an acknowledgement, and the consultation starts.

The workstations provide X-Window based user friendly interfaces and a communication software to transfer consultation framing information. The communication software is based on the TCP/IP stack of ACR-NEMA Digital Imaging and Communications (DICOM) Standards Version 3.0 (ACR-NEMA 1992). The "xv" programs are used for efficient image display on the workstations. Voice software provides the audio/voice interfaces between workstations. A time-stamp mechanism synchronizes image, voice, and annotation commands.

\subsection{Workstation Functional Modules}

A viewing workstation contains three modules: user interface manager, client data server, and remote consultation server. The user interface manager module interfaces with user's queries and provides functions of remote consultation and study session. The client data server communicates with DBMS or DBAS manager through RPCs, sockets, or file transfer for data, image files retrieval and archiving. The remote consultation server module establishes connections between the Local and the Remote workstations before a consultation starts. After the connection is established, the remote consulation server module handles pointing overlay commands as well as voice, video, and image annotation command transfers occurring during the consultation. 


\section{CURRENT PROBLEMS AND SOLUTIONS}

As described above, the Global PACS multimedia data exchanged during remote consultation and diagnosis scenarios are implemented over TCP/IP sockets (Martinez 1995). The communication routines are embedded in both the RCD workstation servers and the DBMS/DBAS managers. In an environment which does not support socket interfaces, it is the user's responsibility to modify the RCD workstation communication routines and the DBMS/DBAS manager to provide the suitable interface.

Moreover, the Global PACS infrastructure is highly heterogeneous. There is no single vendor can provide the best possible solution in every area of Global PACS. It is also necessary to support an integrated software in, for example, advanced multimedia applications for different resources provided by different vendors. The basic client-server mechanism in the current Global PACS architecture will not provide the solution.

In order to increase interoperability of the Global PACS architecture, we have examined the basic properties of object orientation and its applications to heterogeneous, autonomous, and distributed systems. We contend that object-oriented distributed computing is a natural step forward from the current client-server architecture.

Objects form a natural model for a distributed system because distributed components can communicate with each other using only messages addressed through well-defined interfaces. Components are assumed to have their locally defined procedures, enabling them to respond to messages sent to them (Manola 1992). Apparently, the use of objects naturally accommodates the heterogeneity and autonomy of the large-scale Global PACS components: heterogeneity because messages sent to Global PACS components depend only on the components' interfaces, not on their internals; autonomy because Global PACS components can change independently and transparently, provided they maintain their interfaces. In addition, the object-oriented programming carries the Global PACS architecture down to the level where the "components" are individual application abstractions, or even individual data items, rather than the systems or nodes in the Global PACS network.

The Object Request Broker (ORB) defined by the Object Management Group (OMG) provides the interoperability between applications on different machines in a heterogeneous distributed environment. It also seamlessly interconnects multiple object systems. The Common Object Request Broker Architecture (CORBA) illustrates a common desire to connect ORBs and allow interoperation over a wide range of object systems (OMG 1991). Thus, CORBA can support a mix of portable and vendor independent object systems across a number of ORBs.

We present an approach using the CORBA concept to modify and enhance the Global PACS implementation into an object-oriented approach. After mapping Global PACS applications into the CORBA environment, the enhanced Global PACS architecture will provide true multi-vendor interoperability and portability. It also enables the scalability of the system.

\section{GLOBAL PACS IN CORBA ENVIRONMENT}

In CORBA, every system is defined as an object system. CORBA specifies a bare-bones architecture for distributed object management as described before. It includes five inter- 


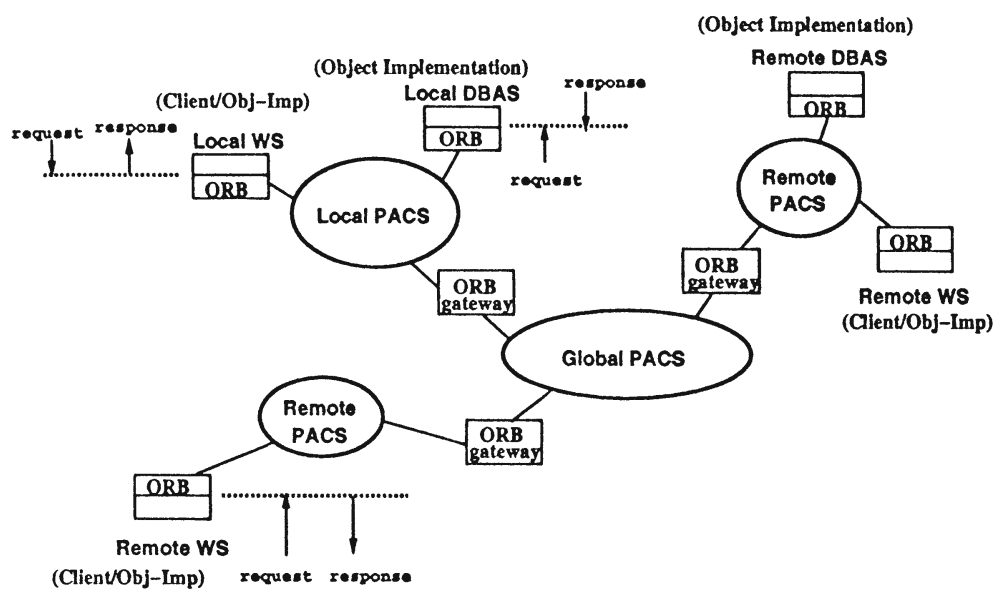

Figure 2 Global PACS CORBA Architecture.

faces and eight major components (Nicol 1993) (OMG 1991). However, at the beginning of the GPACS-CORBA development, we differentiate Global PACS modules into three major components (objects), i.e., the Client, the ORB, and the Object Implementation. The Client is an entity in a RCD workstation, either Local or Remote, e.g., user interface manager that requests services. The Object Implementation is the code and data that actually provides the services. The Object Implementation resides in either the DBAS (DBMS) or RCD workstations depending upon the types of services. The ORB is responsible to find the Object Implementation for the request, to prepare the Object Implementation to receive the request, and to communicate the results back to the request. Thus, DBAS (DBMS) manager, client data server and remote consultation server modules (i.e., network management software) are part of the ORB(s) in GPACS-CORBA environment. In addition, in GPACS-CORBA, the ORB can be implemented as routines resident in the Clients and the Object Implementation, i.e., Client- and Implementation-resident ORB (OMG 1991). In our definition, the Client entity initiates the request by constructing the request dynamically, i.e., Dynamic Invocation Interface. A simplified GPACS-CORBA implementation architecture is shown in Figure 2.

In the diagram, each RCD workstation contains Client, ORB, and Object Implementation components. Each DBAS (DBMS) is composed of Object Implementation and ORB components. A DBAS (DBMS) may have the Client component, if there are requests issued locally. PACSs are connected by ORB gateways. The Client sends requests to the ORB. The ORB finds the right Object Implementation over the GPACS-CORBA networks, possibly through other ORBs and ORB gateway(s). The Object Implementation 
provides the services as requested and sends the responses back to the ORB. Finally, the Client receives the responses as requested.

\subsection{GPACS-CORBA Functional Analysis}

The current Global PACS implementation is developed upon the menu driven functionalities using TCP/IP interfaces for communications. In general, most Global PACS applications are embedded with requests, services, and communication code. However, the requestors of services (i.e., clients) are not isolated from the providers of services. There are no well-defined encapsulated objects and interfaces.

The major issue for GPACS-CORBA development is to identify a minimal set of objects required in the system. In order to define objects, we need to analyze basic functionalities in the system. Despite the functional modules defined in each Global PACS component as presented above, the overall functionalities provided in the Global PACS are:

- access image files to/from DBAS (DBMS)

- access patient, study/event session information to/from DBAS

- perform remote consultation and diagnosis

- quit from the system

These functionalities generate a set of requests. For example,

- image file retrieval/archiving request

- image file displaying request

- establishing remote consultation connection request

- diagnosis framing transfer request during consultation

- patient record retrieval/archiving request

- study/event session retrieval/archiving request

- video/audio interface request during consultation

- quitting request

The services provided for these requests can be categorized as returning an acknowledgement or rejection, an established connection, receiving (or archiving) inquired data, i.e., patient records, study (event) session information, or image files, to/from DBAS, and diagnosis frames exchange between consultation workstations.

According to these requests and services, a detailed mapping of a RCD workstation and a DBAS (DBMS) are provided as follows.

\subsection{RCD Workstation and DBAS in CORBA}

For remote consultation and diagnosis, the Local and the Remote workstations contain functionalities of requesting a connection and establishing the connection. After the connection is established, the Local workstation inquires patient information and image file(s), from a DBAS (DBMS), to be displayed on both the Local and the Remote workstations. The remote consultation starts and annotation exchanges, i.e., diagnosis frames transferring, between the two workstations. The workstations can request the DBAS to store the 


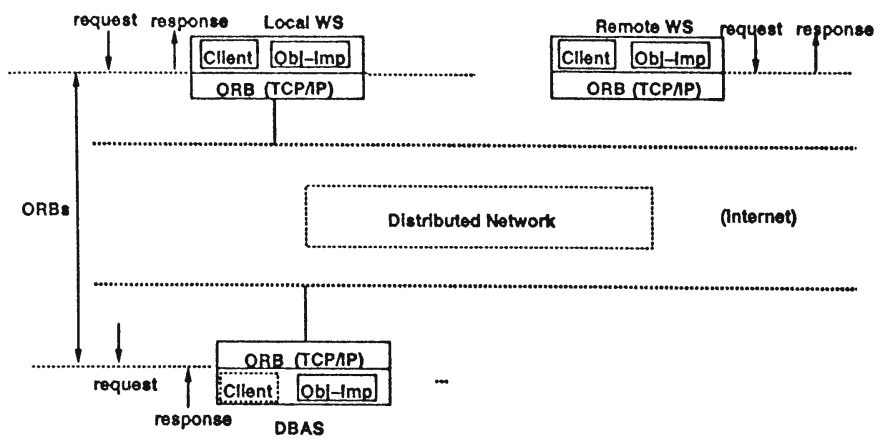

Figure 3 RCD Workstations \& DBAS Architecture in CORBA.

diagnosis session. The DBAS acts as the service provider which responds the requests issued by RCD workstations over the network.

Based on the functionalities presented above, the architecture of workstations for remote consultation and DBAS (DBMS) in CORBA is depicted in Figure 3.

The individual, detailed mapping of RCD workstations and DBAS (DBMS) in CORBA environment are shown in Figure 4 and Figure 5 respectively.

In Figure 4 and Figure 5, when a Local workstation wants to establish a remote consultation session, it constructs a request. For example, an object contains the Remote workstation address, and is sent to the ORB component through its interfaces. It is the ORB's responsibility to find the right Remote workstation and pass the request to it, i.e. the Object Implementation component of the Remote workstation. The Remote workstation processes the request and sends the acknowledgement (i.e., responses) to the ORB. Eventually, the Local workstation will receive the acknowledgement, through the ORB component, sent by the Remote workstation. Now, the remote consultation session is established.

After establishing the connection, the Local workstation constructs a request which contains its address, the DBAS address, the Remote workstation address, and other parameters to inquire a patient's information, image file. The request sends to the ORB component through its interface. The ORB delivers the request to the DBAS. The DBAS acts as the Object Implementation component, which provides patient data management, and displays the image file on both the Local and the Remote workstations.

After the patient's image file displayed on the workstations, the Local workstation constructs a request to exchange diagnosis annotations. The request is delivered to the 


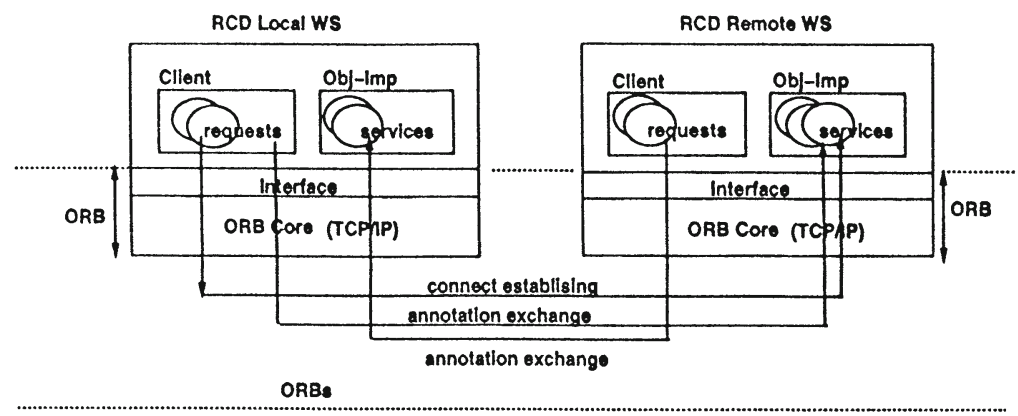

requests: connect request

consultation request

8ervices: acknownledgement

diagnosis framing transfor

Figure 4 RCD Workstations-CORBA Mapping.

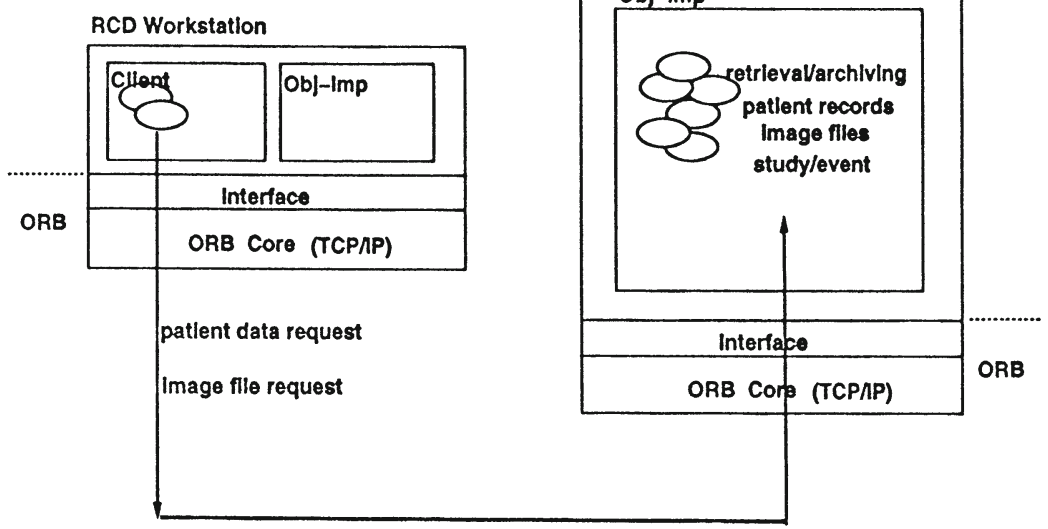

Figure 5 DBAS-CORBA Mapping. 
Object Implementation component of the Remote workstation by $\mathrm{ORB}(\mathrm{s})$. The Remote workstation performs the request and sends the results to the ORB. Eventually, the Local workstation receives the results of the inquiry. Likewise, the Remote workstation can also issue an annotation exchange request, and the Local workstation provides the service.

The RCD workstations consist of Client, ORB, and Object Implementation features in CORBA environment. Similarly, a DBAS (DBMS) contains ORB and Object Implementation components. The ORBs provide the communication mechanism. There can be any communication links as long as they provide the same interface. In other words, in CORBA, the ORB Core is independent of communication links over heterogeneous networks. The Internet (TCP/IP) is the assumed communication mechanism. In addition, the Object Implementation in RCD workstations provides services related to establishing connections and annotation exchanges. The Object Implementation in DBAS (DBMS) provides services associated with patient data management. We can define a single $\mathrm{Ob}-$ ject Implementation component in GPACS-CORBA which combines all of the services provided. However, the "distributed" services over the GPACS-CORBA environment are preferred.

\section{CONCLUSION AND FUTURE WORK}

Initially, the implementation of the GPACS-CORBA architecture is primarily involved the existing Global PACS components not designed to function in the CORBA environment. The integration and migration of Global PACS's components into object-oriented environment are somewhat difficult. However, with the increasing use of object technology within the individual components of Global PACS, (e.g., RCD workstation user interfaces, network management software, database archive system, and operating systems) the boundaries among these components begin to disappear. In addition, tighter integration is possible through the development of standard Global PACS objects, supporting interfaces to the objects and object libraries.

In the GPACS-CORBA implementation, an additional protocol layer is added on top of the usual transport layer protocols (i.e., CORBA over RPC/UDP or TCP/IP). This additional network layer increases the overhead in passing large amounts of data which may cause a significant performance penalty. A light-weight protocol, e.g., XTP (Hutschenreuther 1994), may be introduced into the GPACS-CORBA implementation to increase performance. Moreover, CORBA does not specify how one ORB reveals its naming scheme to others for cross-ORB object interactions (Nicol 1993). It affects GPACS-CORBA interoperability. Therefore, the current GPACS-CORBA development focuses on Internet as the backbone network.

\section{REFERENCES}

Alsafadi, Y., K.M. McNeill, \& R. Martinez (1993). Application driven strategies for efficient transfer of medical images over very high-speed networks, Proc. SPIE Medical Imaging Conference, 14-19 February. 
Association, ACR-NEMA Digital Imaging and Communications Standard: ACR-NEMA Standards Publication No. 300-1992, Washington, D.C.

Hutschenreuther T. et al. (1994) Management Support and Quality of Service Abstractions for XTPX Workshop on New Protocols for Multimedia Systems, pp. 146-154, Berlin, June 29.

Manola, F. et al. (1992) Distributed Object Management, International J. of Intelligent and Cooperative Information Systems, Vol.1, No. 1, pp. 5-42, March.

Martinez, R. et al. (1992) Concepts and system requirements for a Global PACS Proc. SPIE Medical Imaging Conference, pp. 54-63, February.

Martinez, R. et al. (1993) Remote Consultation and Diagnosis in a Global PACS Environment Proc. SPIE Medical Imaging Conference, pp. 296-307, February.

Martinez, R. et al. (1993) Remote Consultation and Diagnosis in Medical Imaging Using a Global PACS Backbone Network Proc. of the European Optical Society, International Symposium on Fiber Optic Networks and Video Communication, April 6, Berlin Germany.

Martinez, R. et al. (1994) Synchronized Voice and Image Annotation in Remote Consultation and Diagnosis for the Global PACS Proc. SPIE Medical Imaging Conference, 13-18 February.

Martinez, R., Y. Alsafadi, and J. Kim (1994) Application of the OSF Distributed Computing Environment to Global PACS Proc. SPIE Medical Imaging Conference, 13-18 February.

Martinez, R., Y. Alsafadi, and J. Kim (1995) Design of Multimedia Global PACS Distributed Computing Environment Proc. Hawaii International Conference on System Sciences - 28, Maui, Hawaii, January.

Martinez, R. et al. (1995) The Rural and Global Medicine Informatics Consortium Network for Radiology Services, Elec. and Comp. Engr. Dept., Univ. of Arizona and Radiology Dept., The Bowman Gray School of Medicine of Wake Forest Univ., in preparation.

Nicol, J. R., T. Wilkes, and F. A. Manola (1993) Object Orientation in Heterogeneous Distributed Computing Systems, IEEE Transaction in Computer, pp. 57-67, June.

Object Management Group (1991) The Common Object Request Broker: Architecture and Specification OMG Document Number 91.12.1, Draft 10 Dec.

Open Software Foundation (1992) Introduction to OSF DCE, Revision 1.0, Prentice Hall, Englewood Cliffs, New Jersey 07632.

Sanders, W. H., R. Martinez, Y. Alsafadi, \& J. Nam (1993) Performance Evaluation of 
a Picture Archiving and Communication Network using Stochastic Activity Networks IEEE Transaction in Medical Imaging.

\section{BIOGRAPHY}

Ralph Martinez is an Associate Professor in the Electrical and Computer Engineering department with Joint Appointments in the Radiology and Pathology department in the College of Medicine. Dr. Martinez has been at the University of Arizona since 1982. Before then, he spent 14 years in industry as a researcher in computer system design and applications, specializing in distributed processing architectures and internet gateways for computer networks.

Since joining the Electrical and Computer Engineering Department, Dr. Martinez has been involved in research in interoperable global information systems, internetworking, picture archiving and communications systems, and multimedia telemedicine systems. His research has been sponsored by the US Army Information Systems Command, AT\&T, Toshiba Medical Systems, and the National Science Foundation.

Sheau-Ling Hsieh is a Ph.D. candidate in the Electrical and Computer Engineering Department, University of Arizona. She was a Principal Engineer and has worked at Intel, Unisys, and Bull. Her work has been focused on networking and telecommunication. 